\title{
Transparent Cross-Layer Solutions for Throughput Boost in Cognitive Radio Networks
}

\author{
Muhammad Faisal Amjad ${ }^{\dagger}$, Baber Aslam ${ }^{\ddagger}$, Cliff Changchun Zou ${ }^{\dagger}$ \\ ${ }^{\dagger}$ Department of Electrical Engineering and Computer Science, University of Central Florida, Orlando FL, USA \\ $¥$ National University of Sciences \& Technology, Islamabad, Pakistan \\ ${ }^{\dagger}\left\{\right.$ faisal, czou\}@cs.ucf.edu ${ }^{\ddagger}$ baber-mcs@ nust.edu.pk
}

\begin{abstract}
Cognitive Radio Network (CRN) is an emerging paradigm that makes use of Dynamic Spectrum Access (DSA) to communicate opportunistically, in the un-licensed Industrial, Scientific and Medical bands or frequency bands otherwise licensed to incumbent users such as TV broadcast. The opportunistic mode of data transfer introduces networkwide quiet periods for spectrum sensing and increased packet losses due to activities by Primary Users. TCP attributes packet delays and losses to congestion in the Internet, which does not perform well in wireless networks where the medium has inherent higher error rates than wired Internet. Opportunistic mode of communication in CRNs aggravates the already error-prone wireless communication resulting in further degradation of TCP performance due to higher latency and decreased throughput. This paper presents an analysis of TCP performance in IEEE 802.22 Wireless Regional Area Network (WRAN) based on Cognitive Radio Networks and proposes two approaches to improve the networking performance. The first approach makes base station resort to local recovery of lost frames between CRN base station and its clients, while the second approach implements a modified split TCP connection in which the base station sends crafted acknowledgements back to an Internet-side host on behalf of the corresponding CRN client to boost transmission speed. To the best of our knowledge, this is the first effort to study TCP performance in IEEE 802.22 based WRANs. Simulation results show that the proposed mechanisms result in improvement in TCP performance by as much as 20 times and conserve bandwidth by reducing retransmission overheads.
\end{abstract}

Keywords: TCP, IEEE 802.22 WRAN, Cognitive Radio Networks.

\section{INTRODUCTION}

A Cognitive Radio Network (CRN) comprises devices that are capable of sensing their radio environment and adjusting operational parameters to communicate in an efficient manner while avoiding any interference with the licensed users of the spectrum, also known as Primary users (PU). The idea of Cognitive Radio (CR) was first presented in [1] which envisioned a $\mathrm{CR}$ as a fully cognitive device capable of observing and adjusting to all possible radio parameters. However recent research has primarily been focused towards spectrum sensing and Dynamic Spectrum Access (DSA) in the TV bands.

Numerous studies have shown that due to the fixed nature of spectrum allocation, large portions of this expensive and very limited resource are being underutilized. One such study in the Chicago metropolitan area [2], carried out between 2008 and 2010 showed that the TV bands' average occupancy was between $30 \%$ and $52 \%$ for the three years with overall average spectrum occupancy for $30-3000 \mathrm{MHz}$ at $14 \%$. In view of this precious yet underutilized spectrum resource, FCC announced that TV channels 5-13 in the VHF band and channels 14-51 in the UHF bands could now be used for broadband Internet access [3] by un-licensed or Secondary Users (SU). It however requires that the SUs, while making use of the spectrum, do not cause interference for any PU's activities. This opportunistic manner of communication opened up numerous exciting challenges to the research community resulting in research initiatives, testbeds e.g. [4] and projects such as the DoD's Joint Tactical Radio System, DARPA's Next Generation (XG) program, IEEE 802.11af also known as White-Fi or super Wi-Fi and IEEE 802.22 Wireless Regional Area network (WRAN) [5], [13].

IEEE 802.22 WRAN is a promising technology which is expected to see widespread deployment especially in the rural areas or areas lacking communications infrastructure. It is designed to operate in TV bands from $54-862 \mathrm{MHz}$ with $47 \mathrm{TV}$ channels. These frequency bands allow long range communication from $17-30 \mathrm{~km}$ with a maximum range of $100 \mathrm{~km}$. The network is organized in a Point-toMultipoint configuration called a Cell with a fixed Base Station (BS) and up to 512 simultaneously associated fixed subscribers or customer premises equipment (CPE) per Cell.

Majority of the research efforts in CRNs have been directed towards the Physical (PHY) and Medium Access (MAC) layers of the protocol stack while the upper layers, especially the transport layer, have not received that much attention. Unlike traditional radio technologies and protocols, much closer interaction among transport layer and the MAC/PHY layers is required in cognitive radio network, mainly because of network-wide quiet periods, opportunistic / dynamic spectrum access, and nondeterministic operation of PUs. These factors can have a devastating impact on the overall throughput and increase retransmission overheads significantly. Facing this challenge, in this paper we present solutions having close interaction between the transport and MAC/PHY layers in order to improve the communication efficiency in a CRN.

Specifically we have made the following contributions in this paper:

- Carried out an analysis of the impact of DSA on TCP performance in the context of an IEEE 802.22 based WRAN (simply called a WRAN from here on). 


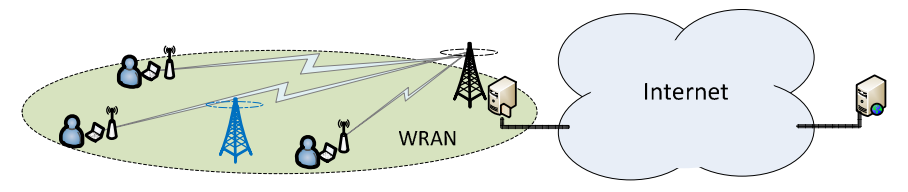

$$
\text { --Primary User --WRAN Base Station }
$$

Figure 1. Network Architecture used for Performance Evaluation and Proposed Solutions

- Identified the factors that result in TCP performance degradation in a WRAN.

- Proposed two schemes to enhance TCP performance.

- Implemented and evaluated the proposed schemes.

The typical architecture of an IEEE 802.22 WRAN is shown in figure-1, which is also the basis for our proposed cross-layer solutions for TCP performance enhancement. In our solutions, the BS which is also the WRAN's gateway to Internet, continuously monitors all TCP traffic and takes appropriate measures to mitigate the impact of PU activity, spectrum sensing and packet losses in the WRAN. Simulation results show that with our proposed schemes, significant performance gains can be achieved in a WRAN while hiding packet losses and delays in the cognitive radio link due to DSA from the sender. In this paper, the terms WRAN and CRN are used interchangeably since we only study WRAN that is built on cognitive radio technology.

Rest of the paper is organized as follows: Section II gives the motivation for our work. Section III presents the proposed solutions for TCP performance boost in WRANs. Some typical results from the simulation of our proposed solutions are given in Section IV, Section V presents related work in the transport layer for CRNs and Section VI concludes the paper.

\section{MOTIVATION FOR CROSS LAYER SOLUTIONS IN CRN}

In this section, we discuss how TCP congestion control algorithm and hence network's throughput may suffer in the context of Cognitive Radio based WRAN. The features of a CRN that could impact TCP performance are: (i) quiet period duration, time that is used for spectrum sensing (ii) the amount of PU activity in the region of a WRAN and (iii) the accuracy of detecting PU's presence by CRN.

\section{A. Quiet Period for Spectrum Sensing}

In order to protect the incumbent PUs from harmful interference, a CRN has to enforce quiet periods in the network to effectively sense the spectrum for its availability. The length of these quiet periods and spectrum sensing can vary depending on the algorithm used [11]. On the other hand, users in a WRAN would expect the same level of QoS (e.g. max delay of $20 \mathrm{msec}$ in case of voice traffic) as in any other network. The IEEE 802.22 WRAN standard therefore, employs a two-stage quiet period management scheme as shown in Figure 2, where the stages are fast sensing $(\sim 9-$ $20 \mu \mathrm{sec})$ and fine sensing $(\sim 0.3-160 \mathrm{msec})$ [17]. The

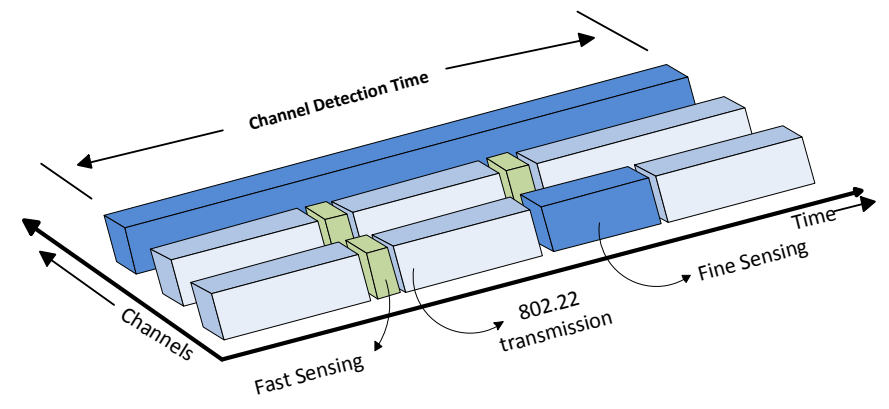

Figure 2. Two-stage spectrum sensing in IEEE 802.22 WRAN

network is synchronized and every time slot $(160 \mathrm{msec})$ is called a Channel Detection Time (CDT). Every CDT can have one or more fast sensing periods and at most one fine sensing period. However, the BS decides to go into fine sensing along with its duration based on the outcome of fast sensing periods. The fine sensing period may occupy a whole time slot. TCP uses RTT measurements for estimating available bandwidth and calculating a suitable Retransmission Timeout (RTO) interval for a particular connection, which is further used in its congestion control algorithm [16]. As per standard TCP Timer management [15], RTO values should be at least $1 \mathrm{sec}$ and implementations must never be more aggressive by selecting smaller values. However, if smaller values of RTO are used e.g. $500 \mathrm{msec}$ in [10] or $200 \mathrm{msec}$ as in [12] as well as Linux, the RTO values will become comparable to WRAN's fine sensing times. In that case, whenever a packet is delayed in WRAN because of fine sensing duration, the source TCP will have a good chance to have premature timeout, erroneously attributing it to congestion and will resort to un-necessary retransmissions, resulting in increased overheads and decreased throughput.

Our simulation study presented in section IV, clearly shows the significant impact of CRN's fine sensing on the performance of TCP communication. For the sake of this paper, we call the probability for BS to decide to conduct fine sensing in a CDT slot as Fine sensing Probability. The simulation results are shown in Figure 3, which shows file transfer completion time (Y-axis) for a file size of $4 \mathrm{MB}$, in an FTP application at three different fine sensing probabilities and for fine sensing durations kept from 0 to $20 \%$ of TCP RTO interval (X-axis).

As concluded in [12], spectrum sensing quiet periods are most debilitating for TCP performance in a DSA network. We believe that a transparent mechanism is therefore needed to monitor the interaction of TCP RTO interval and spectrum sensing quiet periods in the WRAN and take appropriate measures for DSA not to affect TCP congestion control. This would result in boosting TCP throughput while minimizing retransmission overheads and if possible, the end-to-end semantics of TCP connections.

\section{B. Primary User Activity}

The two-stage quiet period and spectrum sensing mechanism of IEEE 802.22 WRAN as shown in figure-1, is intended to strike a balance in ensuring that the PUs are 
protected from harmful interference from the un-licensed

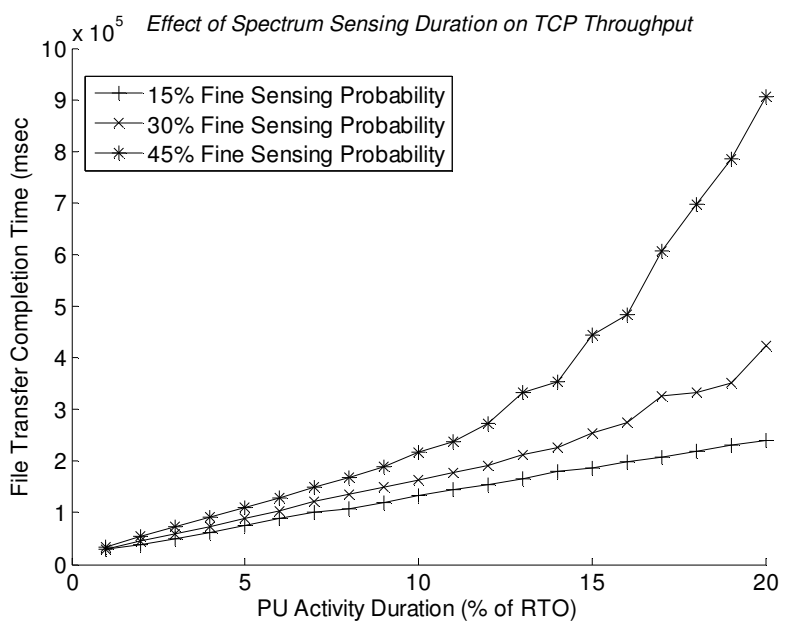

Figure 3. Effects Fine Sensing Probability on TCP Throughput

use of their resource i.e. the spectrum, and efficient utilization of it, in the absence of PUs. During every time slot, called Channel Detection Time (CDT), every node in the WRAN (CPE) carries out fast sensing across a specified number of channels and reports its measurements to the BS. Based on these measurements, the BS may decide not to resort to fine sensing if it concludes that PU's presence on a specific channel is not detected, or it may ask the CPEs to go into fine sensing period, if it suspects presence of a PU and needs further confirmation. Clearly, the two stage sensing mechanism is intended to carry out fine sensing of the spectrum in each CDT slot only if it is necessary, as it would result in the waste of a large chunk of spectrum resource, if the PU was not utilizing the spectrum.

As it is clear that the more the PU activity is observed, the WRAN will spend more of its time in fine sensing of the spectrum. This would result in the network quiet periods adversely affecting TCP RTO calculation and therefore degraded throughput. Simulations have confirmed that as the PU activity grows in the WRAN, nodes spend more and more time in spectrum sensing thereby adversely affecting TCP throughput. Figures 4 and 5 show the performance of TCP under various packet loss rates, fine sensing durations and fine sensing probabilities. For this reason, a mechanism is needed that would monitor the level of PU activity in the WRAN and not let it affect TCP congestion control mechanism.

\section{Primary User Detection Accuracy}

Another factor that will have adverse effects on TCP performance in a $\mathrm{CRN}$ is the accuracy of PU detection scheme(s). On one hand, false alarms in detection of PU's signal will result in wasted spectrum opportunities. On the other hand, if a PU is transmitting in a spectrum band and the CRN is unable to detect its activity, then it will result in harmful interference to the PU as well as packet losses for the CRN. If the probability of not detecting PU's presence is high then there will be significant amount of packet losses in the CRN due to this false spectrum sensing.
In a recent study, probability of PU detection in an IEEE

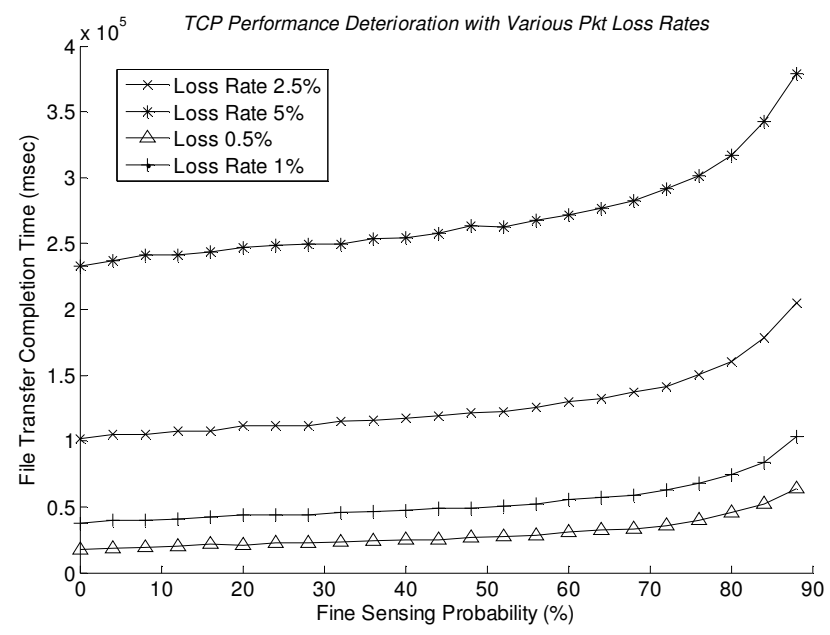

Figure 4. Effects Fine sensing probability on TCP Throughput

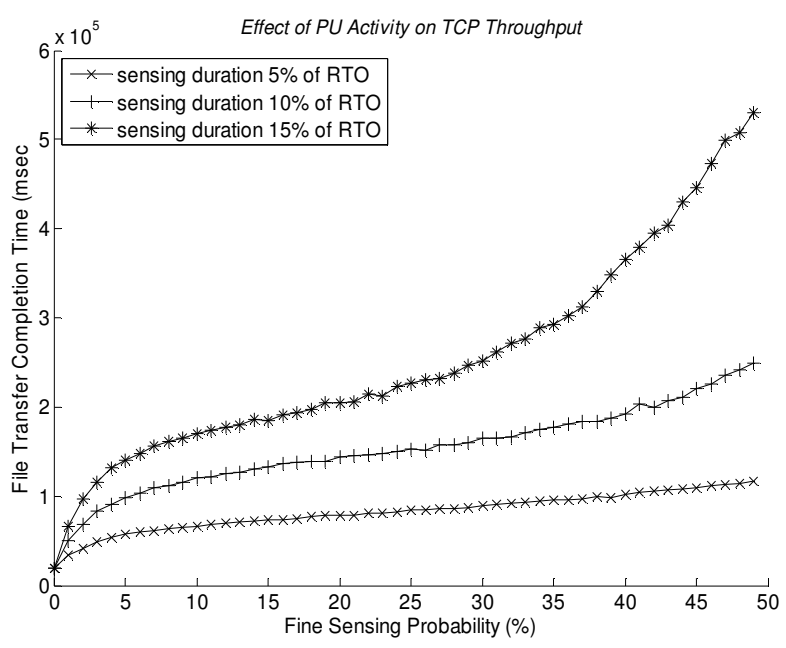

Figure 5. Effects of Sensing Duration on TCP Throughput

802.22 WRAN was found out to be 0.9 while the probability of a false alarm as 0.1 for all signal types [13]. Increase in packet loss probability will further deteriorate the already error prone nature of wireless communications. Therefore, a CRN must have a robust mechanism to recover from packet losses due to spectrum sensing errors.

\section{PROPOSED CROSS LAYER SOLUTIONS FOR PERFORMANCE BOOST IN WRAN}

In this section, we present the details of our proposed solutions for throughput boost in an IEEE 802.22 WRAN. These solutions are transparent because all the proposed enhancements are implemented at the WRAN base station (BS) and no changes are required at source or the destination of a TCP connection. The reasons for this choice are: $(i)$ On one hand, BS is "controller" of all WRAN operations and therefore responsible for scheduling spectrum sensing and usage by CPEs, while on the other hand, it also is the gateway to Internet, as shown in figure- 2 . It therefore has the capability to transparently improve TCP 
throughput. (ii) Implementing enhancements at the BS only, and not at the sender or receiver, resolves the issue of scalability in the context of Internet, and facilitates smooth transition in upgrading BS incrementally. In this section, we first explain the network architecture and then present details of the two proposed solutions for enhancement of TCP performance.

\section{A. Local Loss recovery by Base station}

The first of our proposed solutions to mitigate the effects of increased packet losses and errors in CRN wireless medium is local loss recovery by the WRAN BS. Specifically, the BS continuously monitors all TCP traffic to/from the host(s) in the Internet. It does so by implementing a Loss Recovery Module (LRM) which restricts its actions, which are transparent to both the sender and the receiver, within the context of WRAN only.

The LRM records information regarding all TCP traffic traversing through it in both directions and their associated TCP state which includes sequence numbers and advertised window sizes as well as timers. The LRM restricts the number of un-acknowledged packets sent to the CPE, to a parameter $A D V_{-} W I N$, which represents the current size of the receiver's advertised window. It however, does not implement TCP congestion control mechanism because it has little significance within the context of the one-hop WRAN. For the lifetime of TCP connections, the BS maintains separate duplicate buffers used to maintain copies of all un-acknowledged packets of a particular TCP connection. Packet loss is detected if an acknowledgement ACK is not received before expiration of the local timer or 3 duplicate acknowledgements of a TCP packet are received. Duplicate copy of a lost packet is retransmitted for local recovery of the loss and this is repeated for a maximum of $M A X \_R E T R Y$ times. While any packet loss is recovered locally, any duplicate ACKs from the receiver are dropped at the BS. Values for the retransmission timers at the BS are kept at values selected to ensure that these are large enough so that un-necessary retransmissions are avoided if the ACKs are delayed due to spectrum sensing, but small enough to ensure that packet losses within the WRAN are recovered locally and the sender's RTO interval does not expire. The main advantage of this approach is that the sender's congestion control mechanism does not oscillate un-necessarily, due to losses that occur in the WRAN.

Pseudo-code for the algorithm implemented in LRM is shown in figure-6. The LRM maintains duplicate copies of all TCP packets forwarded to receivers in the WRAN and removes the copies for which ACKs have been received and relayed to the sender in the Internet (lines 10, 11 and 13 of Process_ACKS). It however keeps track of duplicate ACKs and if 3 duplicate ACKs are received or its local timer expires, it is treated as an indication of a lost packet resulting in appropriate packets being retransmitted out of duplicate buffer. Sending of new or duplicate packets is kept within the limit of advertised window size of the receiver (lines 4, 7, 9 of BS_Process). The Fast Retransmit option enables the BS to recover from a packet loss without the expiry of its local timer.

\section{B. Split TCP Connection}

Our second approach to boost TCP throughput for data transfers across a WRAN is to implement what we call a split TCP. Differences between existing split TCP mechanisms [19], [20], [21] and our approach are

- Our approach does not require modifications in either the source or the destination;

- It is specifically designed for IEEE 802.22 WRANs;

- It pre-acknowledges TCP packets to shield the sender from unusually large spectrum sensing delays.

The BS implements this scheme with a module similar to LRM, which Pre-Acknowledges TCP packets received at the BS, on behalf of the receiver in WRAN, effectively sending spoofed ACKs to the sender. This module is called the Pre-Acknowledgement Module (PAM). PAM maintains the state of all TCP connections traversing the BS, and just as the LRM, maintains a duplicate buffer for every TCP connection to ensure delivery of all of its packets. PAM also does not implement the complete TCP and its congestion

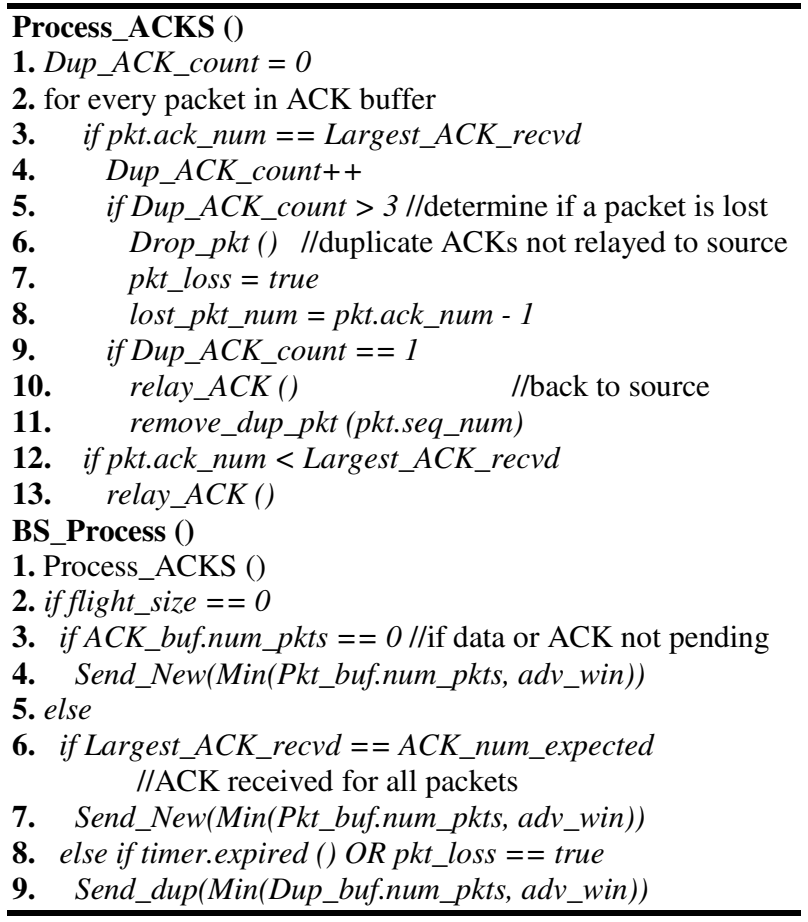

Figure 6. Pseudo-code for LRM algorithm at Base Station

control mechanism; however it restricts the number of packets transmitted to the receiver's advertised window size along with local timers for reliable delivery. Unlike LRM, values for PAM's retransmission timers are not associated with the sender's RTO interval because in this case the sender's transmissions are independent of losses or delays in the WRAN. Therefore, PAM's local timers can be configured to suit the requirements of WRAN. 


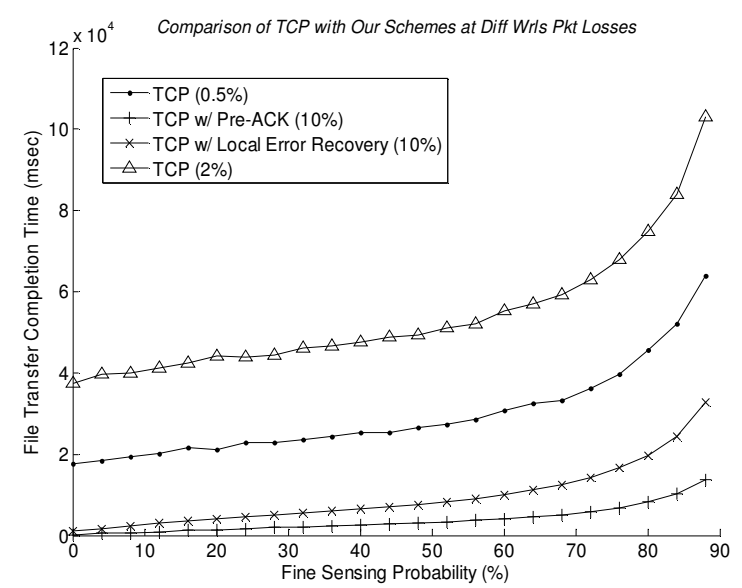

Figure-7. Performance of Proposed Solutions at various Loss

Algorithm of PAM is similar to LRM as shown in figure-6, with following exceptions: (i) whenever new packets are received at the BS from a sender in the Internet, an Acknowledgement is immediately sent to sender, and (ii) when an ACK is received from the receiver in WRAN, it is dropped and corresponding duplicate packets are dropped from duplicate buffer. Packet retransmissions out of duplicate buffer are triggered when the BS's local timer expires or with the receipt of 3 duplicate acknowledgements to trigger Fast Retransmission.

\section{Discussion and limitations of proposed approaches}

The split TCP connection approach for boosting TCP performance uses spoofed acknowledgements by the BS which essentially breaks the end-to-end semantics of a TCP connection since a packet is acknowledged to its sender whereas it might not have been received by its intended receiver. This situation may not be acceptable to certain applications that work on the guarantees from TCP regarding actual delivery of a packet that is acknowledged. However, in situations where TCP RTO interval is comparable to fine sensing duration of the WRAN, preacknowledgement might be the only option for preventing the TCP congestion control algorithm to timeout repeatedly and incorrectly attributing it to congestion. On the other hand, local recovery of lost packets by the BS preserves end-to-end semantics of TCP connections and provides a throughput boost as well. Therefore, the solution with preacknowledgements by BS may be selected for applications such as file transfer that may not be concerned with preserving end-to-end semantics while local loss recovery may be selected for the rest.

The main advantage of our schemes is that they provide throughput boost transparently i.e. without any need for changes in the end systems making them ideal for initial deployment of a WRAN.

\section{PERFORMANCE EVALUATION}

Simulation Setup: In this section, we evaluate the performance of our proposed solutions to boost TCP throughput in the context of a WRAN. Specifically, we consider a file transfer application in which a node in an IEEE 802.22 WRAN communicates with an FTP server in

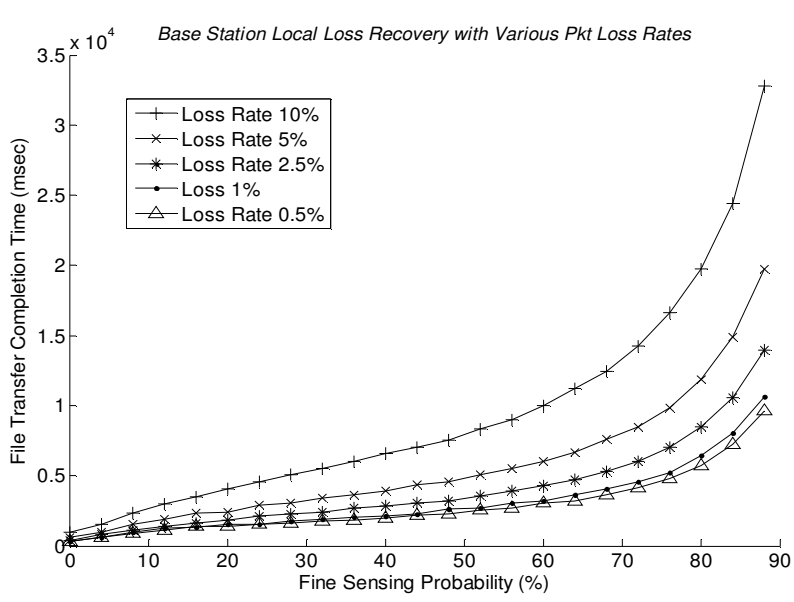

Figure-8. Performance of Local Loss Recovery at various Loss

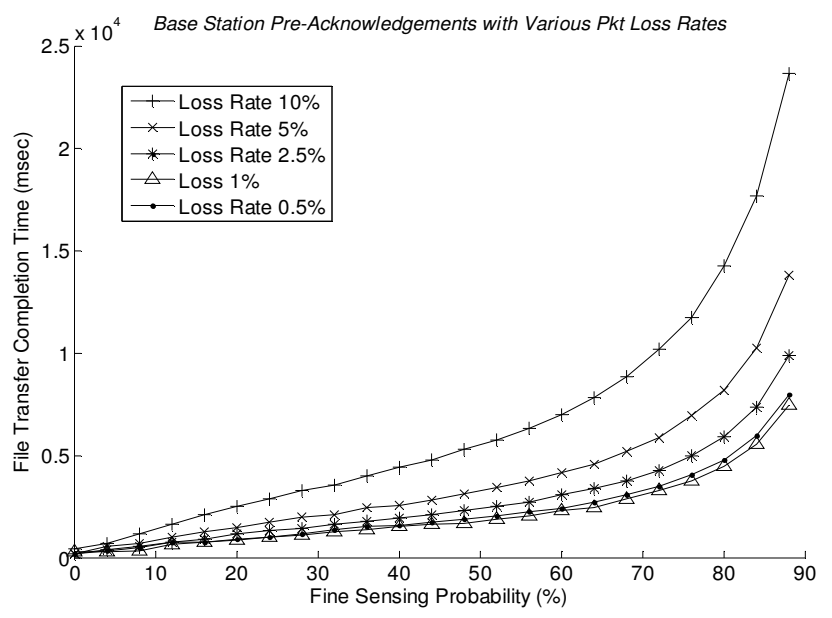

Figure-9. Performance of Split TCP (Pre-ACK) at various Loss Rates

the Internet. We have developed a simulator to model a WRAN in which the BS and its associated CPEs resort to DSA to communicate with each other while the BS acts as gateway to Internet for the WRAN. Every node (CPE) in the network is equipped with a single radio transceiver that can be tuned to one channel at a time, however all nodes in the network are tuned to the same channel and the use or switching to/from a specific channel is controlled by the BS. The TCP module of our simulator implements slow start, congestion avoidance and fast retransmission schemes. However, since there have been various values for RTO interval for TCP implementations, we have also studied the impact of varying its values in our simulations. The downlink bandwidth from BS to CPEs is $1.5 \mathrm{Mbps}$ downlink and uplink bandwidth per CPE is 384 kbps [13], [17]. The receiver of a TCP packet never delays an outgoing ACK for piggybacking i.e. it sends one ACK for every packet received. Bandwidth between the WRAN BS and FTP server is assumed to be $10 \mathrm{Mbps}$ and file size for FTP transfer is $4 \mathrm{MB}$. Measurements for every data point were recorded by averaging the results of 1000 simulation runs.

Figure-7 shows the performance of our proposed solutions in comparison with TCP where the figure shows TCP performance at packet loss rates of $0.5 \%$ and $2 \%$ for the WRAN link whereas our proposed schemes were run 


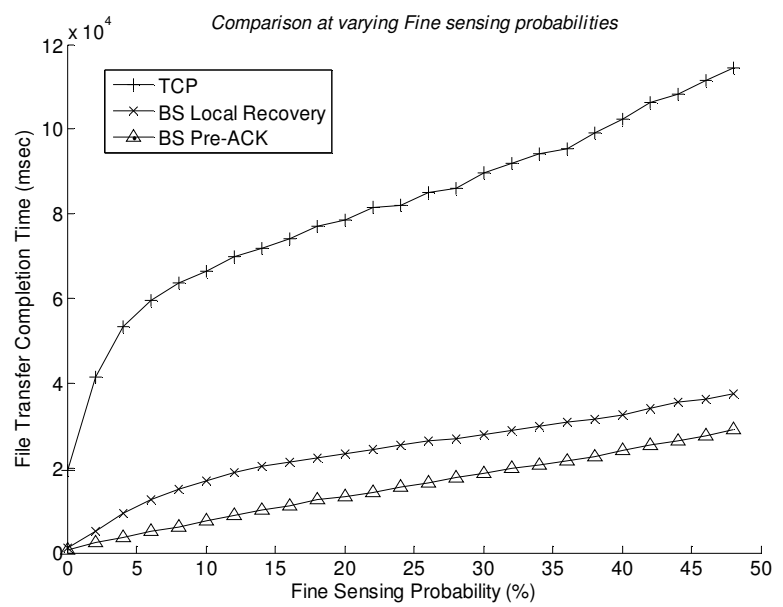

Figure-10. Performance comparison at Fine Sensing Duration 5\% of TCP

with $10 \%$ packet loss rate in the WRAN. As it is evident, our proposed schemes increase the throughput by 20 times. Figures 8 and 9 show the performance of our proposed schemes individually where the simulations were run for packet loss rates from $0.5 \%$ to $10 \%$ and the overall file transfer times (y-axis) were recorded against varying fine sensing probabilities (x-axis) form 0 to $90 \%$. The difference in performance of local loss recovery and preacknowledgement schemes can be observed because in case of local recovery, the sender does not send new packets unless the losses in WRAN have been recovered by the BS, whereas for pre-acknowledgement, the sender can send new packets at the full capacity of the Internet link and is not restricted due to losses and delays in the WRAN. In this way, losses in the WRAN have some impact on the overall throughput of local loss recovery mechanism but none for pre-acknowledgement scheme.

Figures 10 and 11 show a comparison of TCP's performance with our proposed solutions, at fine sensing durations $5 \%$ and $15 \%$ of TCP RTO intervals respectively, where the packet loss rate is kept constant at $0.2 \%$ for WRAN and $0.01 \%$ for Internet. In addition to preventing the fine sensing periods to interfere with TCP RTO interval, our schemes provide better throughput even when there are no fine sensing periods, because they are also able to recover from packet losses without having the sender TCP being affected by them.

Our proposed solutions perform up to 20 times better than TCP in scenarios where TCP flows suffer heavy packet losses and the sender has to invoke its congestion control mechanism very frequently. On the other hand, our solutions shield the sender from adverse effects of losses in the WRAN. When compared with each other, the two proposed solutions exhibit different performance as well. Split TCP connection with Pre-Acknowledgements has better performance than the local loss recovery by BS, but may be a less favorable option when it comes to preserving the end-to-end semantics of a TCP connection. However, we propose that the choice of selecting a transfer mechanism other than TCP, be negotiated by CPEs with the

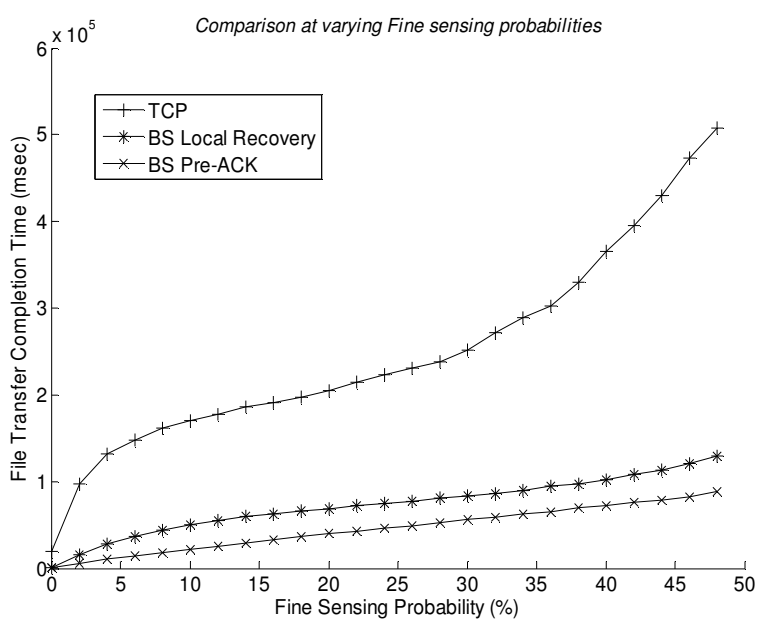

Figure-11. Performance comparison at Fine Sensing Duration 15\% of TCP

$\mathrm{BS}$ at the time of joining the WRAN or for specific TCP flows.

\section{RELATED WORK}

Researchers have only recently started to lay down the requirements for CRNs [6], [7] and novel platforms e.g. [8] are being developed for advanced research in software defined cognitive radios. Development of transport layer protocols for CRNs is largely an un-explored area with a few exceptions.

TP-CRAHN [9] is a protocol designed for use within $a d$ hoc CRNs. It incorporates a cross-layer design with explicit feedback from every node between the source and destination nodes, regarding their sensing schedules and the length of quiet periods intended for detecting PUs through spectrum sensing. Its transport protocol also interacts with physical layer's channel information, link layer's buffer management as well as a mobility prediction framework to cater for varying parameters and network dynamics. TCPE [10] protocol is designed for heterogeneous networks involving CRNs and the wired Internet. It aims to achieve better throughput through available bandwidth estimation and round-trip-time (RTT) difference between successive TCP segments. It assumes packet delays to have been caused by spectrum sensing if the difference between successive RTTs is more than $90 \%$ of spectrum sensing duration (SSD). The study does not present any results if the source is in the Internet and destination node is located in a CRN, in which case the source node would have to know the SSD for destination node's network, which can vary by technology [11].

An approach similar to ours, based on local loss recovery, has been employed in [18], but the main difference from our approach is that the above scheme is designed for Cellular networks to mitigate the effects of losses due to high bit-errors and handoffs whereas our scheme is specifically designed to cater for additional packet losses and delays that occur due to DSA. Unlike [18], our scheme does not require the use of Explicit Congestion Notifications (ECN) or Negative Acknowledgements (NACK). If a sender is located in the 
WRAN, then it would be aware of spectrum sensing schedules and its associated delays and would therefore be able to discern packet losses or delays due to quiet periods from those caused due to factors other than DSA. On the other hand, if the sender is in the Internet and packets arrive successfully at BS but losses or delays occur within the WRAN due to DSA, then there is no need to use ECN or NACK with the sender when the loss can be recovered locally by BS. The use of ECN or NACKs is therefore of little use in the context of DSA which would also require modifications to both the source and the destination, thereby introducing the problem of scalability of the solution.

I-TCP [19] was proposed to split a TCP connection at the BS into two separate connections, to alleviate TCP poor performance in wireless networks. It however required special devices called Mobility Support Routers in the wireless network and also required modification to the TCP code in mobile hosts. In [20], a split connection approach is proposed for ad hoc cognitive networks to mitigate the effects of TCP un-fairness towards nodes that are relatively farther away from the source node. It works by forming a chain of suitably selected nodes to act as TCP proxies between the source and destination of a connection, both of which lie within the ad hoc network. A Semi-Split TCP has been proposed in [21], in which a BS never preacknowledges a packet, rather it buffers the ACKs received from the receiver in cognitive network and controls the amount of ACKs relayed back to the sender in order to throttle the sender and to prevent the BS's buffers from starvation.

Performance analysis of various flavors of TCP in DSA links is presented in [12]. The study concludes that the most dominant factor in TCP throughput reduction is spectrum sensing duration and not the PU detection errors.

\section{CONCLUSION}

In this paper we have shown that TCP performance is affected adversely due to PU activity in a WRAN, DSA by the un-licensed users and associated quiet periods that can be comparable with RTO interval of the sender's TCP, a scenario which can trigger TCP's congestion control mechanism un-necessarily and fruitlessly. We have shown that our solutions transparently remedy this situation by coping well with packet losses that are inherent to the wireless medium as well as resulting from inaccuracies in detection of PUs and network-wide quiet periods for spectrum sensing. To the best of our knowledge, there is however, no work that analyses TCP performance in the context of an IEEE 802.22 WRAN, or proposes solutions that would result in throughput boost for these networks. Currently we are investigating the impact of DSA in a multi-hop cognitive radio network such as IEEE 802.11af.

\section{REFERENCES}

[1] Mitola, J. III, Maguire, G.Q Jr, "Cognitive radio: making software radios more personal," IEEE Personal Communications, vol.6, no.4, pp.13-18, Aug 1999.
[2] Taher, T.M, Bacchus, R.B, Zdunek, K.J.; Roberson, D.A, "Long-term spectral occupancy findings in Chicago," New Frontiers in Dynamic Spectrum Access Networks (DySPAN) 2011.

[3] U.S. FCC, ET Docket 04-186, "Notice of Proposed Rule Making, in the matter of Unlicensed Operation in the TV Broadcast Bands," May 25, 2004.

[4] Mishra, S.M. Cabric, D. Chang, C. Willkomm, D. van Schewick, B. Wolisz, S. Brodersen, B.W. , "A real time cognitive radio testbed for physical and link layer experiments," New Frontiers in Dynamic Spectrum Access Networks, DySPAN 2005

[5] IEEE 802.22 2011 Standard for Wireless Regional Area Networks in TV Whitespaces, http://www.iee.org/22

[6] Nekovee, M. , "A survey of cognitive radio access to TV White Spaces," ICUMT 2009.

[7] Ian F. Akyildiz, Won-Yeol Lee, and Kaushik R. Chowdhury. "CRAHNs: Cognitive radio ad hoc networks". Ad Hoc Networks, 2009.

[8] Minden, G.J, Evans, J.B, Searl, L, DePardo, D, Petty, V.R, Rajbanshi, R, Newman, T, Chen, Q, Weidling, F, Guffey, J, Datla, D, Barker, B, Peck, M, Cordill, B, Wyglinski, A.M, Agah, A, "KUAR: A Flexible Software-Defined Radio Development Platform," New Frontiers in Dynamic Spectrum Access Networks, DySPAN 2007.

[9] Chowdhury, K.R, Di Felice, M, Akyildiz, I.F, "TP-CRAHN: a Transport Protocol for Cognitive Radio Ad-Hoc Networks," INFOCOM IEEE Conference on Computer Communications, 2009.

[10] Sarkar, D, Narayan, H, "Transport Layer Protocols for Cognitive Networks," INFOCOM IEEE Conference on Computer Communications, 2010.

[11] Yucek, T, Arslan, H, "A survey of spectrum sensing algorithms for cognitive radio applications," Communications Surveys \& Tutorials, IEEE , vol.11, pp.116-130, 2009.

[12] Slingerland, A.M.R, et. Al. "Performance of Transport Control Protocol Over Dynamic Spectrum Access Links," New Frontiers in Dynamic Spectrum Access Networks, DySPAN 2007.

[13] Stevenson, C, et.al, W, "IEEE 802.22: The first cognitive radio wireless regional area network standard," IEEE Communications Magazine, vol.47, pp.130-138, 2009.

[14] Cordeiro, C, Challapali, K, Birru, D, Sai Shankar, N, "IEEE 802.22: the first worldwide wireless standard based on cognitive radios," New Frontiers in Dynamic Spectrum Access Networks,. DySPAN 2005.

[15] Allman, M, Paxson, V, "Computing TCP Retransmission Timer", RFC 2988, November 2000.

[16] Allman, M, Paxson, V, Blanton, E, "TCP Congestion Control", RFC 5681, September 2009.

[17] IEEE 802.22 Draftv3.0 -Members only Documents of the IEEE 802.22 Working Group.

[18] Balakrishnan, H, Seshan, S, Katz,R.H, "Improving reliable transport and handoff performance in cellular wireless networks", Wireless Networks Journal 1995.

[19] Bakre, A. Badrinath, B.R., "I-TCP: indirect TCP for mobile hosts," 15th International Conference on Distributed Computing Systems, 1995.

[20] Kopparty, S. Krishnamurthy, S.V. Faloutsos, M. Tripathi, S.K., "Split TCP for mobile ad hoc networks," IEEE Globecom 2002.

[21] Xie, F, Jiang N, Hua, Y. H, Hua, K.A., "Semi-Split TCP: Maintaining End-to-End Semantics for Split TCP," IEEE LCN 2007. 